\title{
Detection of QTLs controlling pollen germination and growth in maize
}

\author{
M. SARI-GORLA*, M. E. PÉ \& L. ROSSINI \\ Department of Genetics and Microbiology, University of Milano, Via Celoria 26, 20133 Milano, Italy
}

\begin{abstract}
Pollen competitive ability, which represents the reproductive success of a pollen grain, is the result of various pre- and post-shedding components. The genes controlling two of these, pollen germination and tube elongation, measured in artificial conditions, were identified and their chromosomal localization established by RFLP analysis in a population of recombinant inbred lines. Six regions were detected for pollen grain germination and seven for tube growth, which explained 45 per cent and 70 per cent, respectively, of the phenotypical variability of the traits. The results, interpreted together with those relating to in vivo germinability and tube growth rate, obtained in a previous experiment, suggested the existence of a system of timing of different sets of genes controlling the gametophytic developmental stages.
\end{abstract}

Keywords: maize, pollen germination, QTL mapping, RFLP analysis, tube growth.

\section{Introduction}

The biological significance of pollen competitive ability (PCA), which describes the reproductive success of a pollen grain, derives from the fact that pollen fitness is a component of plant fitness; pollen selection and response to selection in the sporophytic progeny have been demonstrated in different species, with regard to traits such as plant vigour, fertility and tolerance to stresses (Ottaviano \& Mulcahy, 1989; Hormaza \& Herrero, 1992).

PCA is the result of various pre- and post-shedding components; among the former are viability and competition within the anther, due to differences of metabolic efficiency during microspore development; among the latter are germination time and rate, tube growth rate and fertilization ability.

The detection of stage-specific genes controlling gametophytic development and function suggests that PCA is controlled by diverse genetic systems. Differential effects of specific chromosomal deficiencies on the development of maize pollen grains have been demonstrated by means of B-A translocations (Kindiger et al., 1991), that allow the production of aneuploid pollen in which a portion of the microspores are missing a chromosome arm. The diverse deficiencies, according to the chromosome fragment lost, showed that components of normal microspore development are con-

*Correspondence. trolled by genes located in a specific part of the genome. Studying monogenic embryo-lethal mutants in Arabidopsis (Meinke, 1982) and defective-endosperm mutants in maize (Ottaviano et al., 1988), genes affecting both kernel development and/or pollen function were identified. The analysis of the progeny produced by heterozygous plants showed a significant distortion in the Mendelian segregation rates, indicating different fertilization ability between normal pollen and pollen carrying the mutation.

Moreover, the outcome of pollen competition can be modulated by the stylar tissue genotype, which can act as a sieve with regard to pollen tubes competing to achieve fertilization. Even though precise data concerning the biological basis of pollen-style interaction are very scarce, it is reasonable to think that it concerns the metabolic efficiency of pollen genotypes in taking up nutrients supplied by the female counterpart (Pfahler, 1967; Sari-Gorla et al., 1976; Linskens \& Pfahler, 1977).

In a previous experiment, we identified and localized on maize chromosomes the quantitative trait loci (QTLs) mainly responsible for the variability of two PCA components: (i) germinability and early pollen tube growth, and (ii) late tube growth rate, up to fertilization, measured in vivo. The data confirmed that many loci are involved in the control of the traits and suggested that different sets of genes are involved in the control of the two components (Sari-Gorla et al., 1992). 
In order to complete the picture of the genetic control of PCA components, including those certainly not affected by interactions with the stylar tissues, RFLP analysis was used to study pollen grain germination in vitro and tube elongation after 3 hours of growth on artificial medium.

\section{Materials and methods}

The plant material was the same RI population, obtained from the cross $\mathrm{T} 232 \times \mathrm{CM} 37$ and typed for about 200 RFLP loci at a known localization (kindly furnished by B. Burr) already used for in vivo PCA component evaluation (Sari-Gorla et al., 1992).

Freshly shed pollen from field plants was grown on artificial medium $\left(100 \mathrm{mg} \mathrm{H}_{3} \mathrm{BO}_{3}, 300 \mathrm{mg} \mathrm{Ca}\left(\mathrm{NO}_{3}\right)_{2}\right.$, $170 \mathrm{~g}$ sucrose, $7 \mathrm{~g}$ agar/1), solidified in a glass cylinder, cut in slices of 1-2 mm and put in Petri dishes. For each line, two replicates were made in 2 days. After $3 \mathrm{~h}$ at $27^{\circ} \mathrm{C}$, the dishes were fixed with Farmer's liquid and stained with Aniline blue. For trait evaluation, the dishes were divided into eight sectors, in each of which 50 grains were scored for germination percentage under a binocular dissection microscope and 50 tubes per plate were measured by means of a graphic digitizer (Video plan).

The detection of the chromosome regions where QTLs controlling the characters studied are located, was carried out by regression analysis, where the mean value of the character for each line is the regressed variable and the allelic composition of the molecular marker is the regressor, assuming the value of 1,2 or 1.5 , according to the presence of the alleles from the first parental line, from the second parental line, or from both (heterozygous locus). A significant regression is interpreted as an indication that a QTL controlling the trait is closely linked to that RFLP locus.

To avoid false assignments, the correlation matrices between all the restriction loci giving a significant result were computed. The cumulative contribution of the loci to the character variability was evaluated including in a multiple regression model all the significant, uncorrelated loci. The number of significantly associated loci identified is thus a conservative minimum.

\section{Results}

Figure 1 shows the position on maize chromosomes of the loci significantly associated with QTLs controlling the two characters; the nine loci associated with pollen grain germination (PGG) are marked with a circle and the 21 associated with tube growth (PTG) are marked with an asterisk; the bars indicate the coefficient of determination, $R^{2}$, which measures the amount of trait variability explained by its association with that molecular locus.

The real number of loci involved in the control of the traits is likely to be fewer. In fact, a cluster of signifi-
Fig. 1 Chromosomal localization of RFLPs significantly associated with QTLs controlling in vitro pollen germination (circle) and pollen tube growth (asterisks). Bars represent the degree of correlation between RFLP and pollen traits.

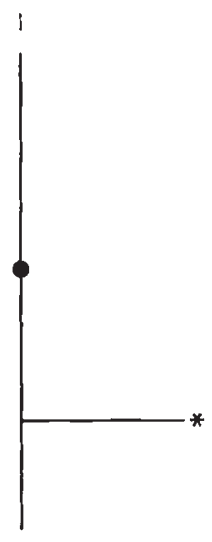

6

2
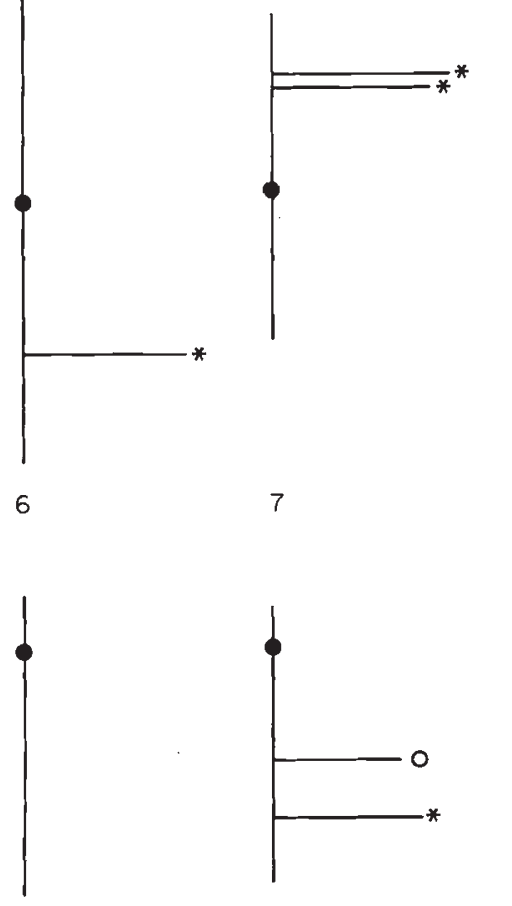

3
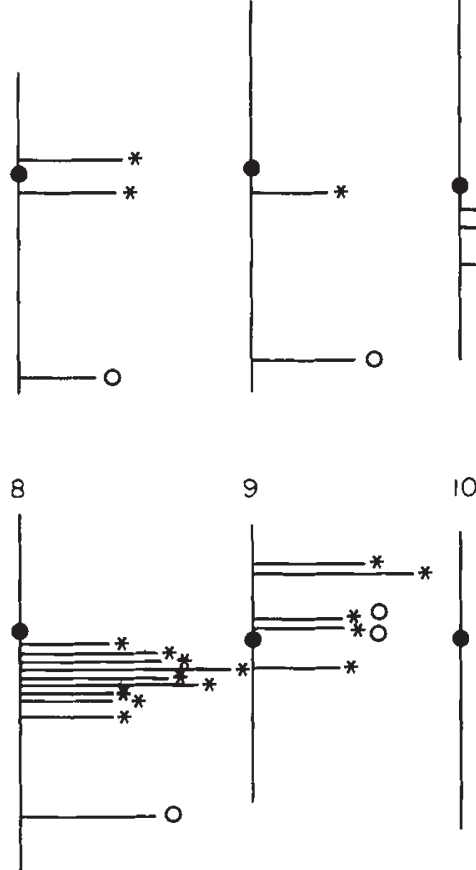

5

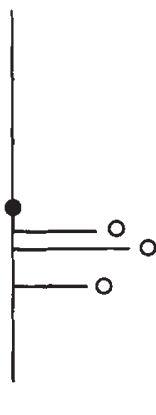

10 
cant loci could be the result of the association between the QTL and the adjacent molecular loci. Moreover, sampling effects during the inbreeding process can result in apparent linkage between restriction loci.

Taking into account these points, the chromosomal regions in which at least one QTL involved in the control of the traits is located were identified on the basis of the correlation matrices between molecular markers. For each cluster of significantly correlated loci, only that having the highest $R^{2}$ was chosen; the analysis indicated six and seven loci as being responsible for PGG variability and PTG variability, respectively; the values of $R^{2}$, obtained by including all the significant and uncorrelated loci for each trait in a multiple regression model, were 0.46 and 0.70 , respectively, indicating that a large part of the phenotypic variability of the traits is accounted for by QTLs detected by the analysis.

Table 1 reports the RFLP loci significantly associated with the in vitro component of PCA, pollen grain germination and tube growth rate. The corresponding coefficient of determination and coefficient of regression are indicated; the latter represents the estimated effect: its numerical value measures the effect of the allelic substitution and its sign indicates the allelic distribution in the two parental lines.

The data reveal that only one identified chromosomal region carrying QTLs is common to PGG and PTG; the other five and six molecular markers, respectively, appear to be linked to QTLs that specifically affect one or the other of the characters.

Table 1 RFLP loci showing significant effects on in vitro pollen grain germination and pollen tube growth

\begin{tabular}{|c|c|c|c|c|c|}
\hline \multirow[b]{2}{*}{ Chromosome } & \multirow[b]{2}{*}{ Locus } & \multicolumn{2}{|c|}{$\begin{array}{l}\text { Pollen grain } \\
\text { germination }\end{array}$} & \multicolumn{2}{|c|}{$\begin{array}{l}\text { Pollen tube } \\
\text { growth }\end{array}$} \\
\hline & & $R^{2}$ & $b$ & $R^{2}$ & $b$ \\
\hline 1 & $B z 2$ & & & 0.20 & 0.23 \\
\hline 2 & NPI239 & & & 0.22 & 0.60 \\
\hline 3 & $\begin{array}{c}B N L 8.35 \\
N P I 425\end{array}$ & 0.09 & -3.61 & 0.13 & 0.41 \\
\hline 4 & $\begin{array}{c}B N L 15.27 \\
B N L 8.23\end{array}$ & 0.14 & 15.91 & 0.09 & -0.32 \\
\hline 5 & $B N L 10.12 L$ & 0.15 & -15.15 & & \\
\hline 7 & $\begin{array}{c}B N L 8.37 \\
N P I 113\end{array}$ & 0.18 & -17.35 & 0.15 & -0.44 \\
\hline 8 & $\begin{array}{c}B N L 9.44 L \\
B N L 10.24 B\end{array}$ & 0.16 & -17.17 & 0.29 & -0.71 \\
\hline 9 & BNL3.06 & 0.12 & 15.14 & 0.12 & 0.42 \\
\hline
\end{tabular}

$R^{2}$ : coefficient of determination; $b$ : estimated effect.

\section{Discussion}

The use of molecular markers proved to be an efficient way to localize QTLs involved in the control of pollen germination and pollen tube growth as evaluated in vitro. By means of regression analysis we detected a minimum number of six and seven chromosomal regions carrying genes controlling in vitro $\mathrm{PGG}$ and in vitro PTG, respectively. Even though the nature of the estimate is only indirect, our findings strongly confirm the complex basis of the genetic system that regulates pollen function.

Furthermore, the observation that only one QTL is involved in the control of both traits, leads us to postulate the existence of two distinct sets of genes, one controlling germination rate, the other active during pollen tube growth.

A possible physiological interpretation of our results can be made by comparing these findings and those previously obtained after the characterization of the same RI population for the component of PCA measured in vivo (Sari-Gorla et al., 1992). This comparison of the two studies (Fig. 2) reveals that four QTLs, accounting, in this study, for about 40 per cent of the phenotypic variability of in vitro PTG, are also significantly associated with in vivo germination and early tube growth, whereas all the identified chromosomal regions carrying QTLs for (in vivo) later tube growth rate within the style appear to be specific for this trait.

Bearing in mind that the growth of maize pollen tubes in artificial medium is very limited, $1-2 \mathrm{~mm}$, compared with in vivo growth, where, according to the position of the ovule along the ear, the pollen tube can grow up to $20-30 \mathrm{~cm}$ to accomplish fertilization, the combination of our recent and previous results suggests the existence of three different sets of genes expressed during the various stages of the fertilization processes. A first group of genes affecting germination rate, evaluated in vitro, is active during microsporo-

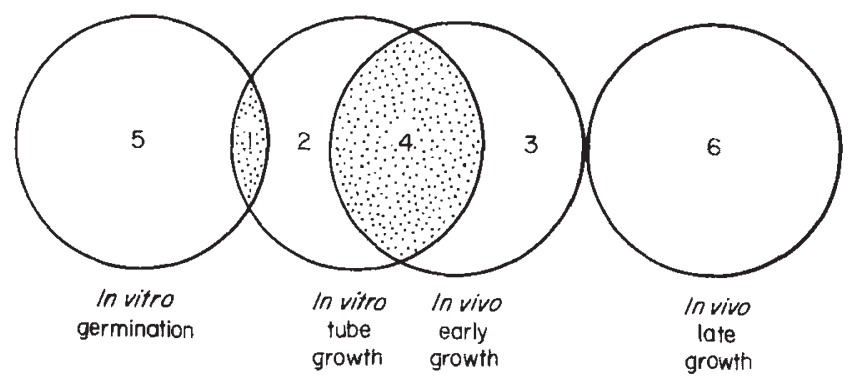

Fig. 2 Association between molecular markers and different pollen functions. Data for in vivo components are from SariGorla et al.(1992). The number of specific or common loci is indicated. 
genesis. A second set controls the early phases of tube lengthening, and thus is responsible for variability of in vitro tube elongation and in vivo early growth. Finally, other genes regulate pollen tube growth when the tube is well established into the style, up to fertilization.

This interpretation agrees with what is known about pollen development physiology: different functions are involved during pollen germination and tube elongation up to fertilization. In the first two stages, enzymes are used and reserve materials that are already present in the grain are mobilized (Mascarenhas, 1990) whereas during pollen tube growth into the style, other processes are activated, involving interaction between growing pollen and the stylar tissues of the female plant.

The results confirm what has been observed with regard to the PCA components evaluated in vivo: early tube growth, expressed both in vitro and in vivo, appeared to be negatively correlated with later tube growth rate into the style. It is possible that the same locus has an effect on two different traits, where one allele promotes the first and the other allele promotes the second. On the other hand, it can be supposed that alleles favouring early stages of pollen function and later phases of growth are closely linked in trans, so that an allele increasing the first component and one decreasing the second are inherited together.

In Viola, a negative correlation between germination time and tube growth rate has been observed (Dajoz et al., 1992), according to the number of pollen apertures: pollen with four apertures germinates quickly but has a slow tube growth, pollen with three apertures germinates slowly but grows quickly. In this case, it is possible to hypothesize that a fast germination rate is obtained by consuming reserve materials which thus become unavailable for subsequent tube growth, whereas the slow germination pollen preserves the materials to be used during the subsequent phase of growth. This is not expected to be the case in maize pollen where, due to the disproportion between pollen grain diameter and tube length (about $90 \mu \mathrm{m}$ and 20 $\mathrm{cm}$, respectively), the late pollen tube growth is mainly sustained by the metabolic support of the female tissues.

Moreover, possible differences in pollen diameter, affecting the two characters in opposite ways, could not be invoked as this character did not reveal genetic variation in the studied population and was also quite homogeneous within lines (data not given).

An interpretation of these findings should take into account the strategy of the sporophyte: it is possible that linkage is maintained because of an adaptive genetic combination for sporophytic fitness. In any case, as these observations relate to segregating genes from only two lines, the parents of the RI population, they obviously do not represent a typical situation of the maize germplasm; this aspect will possibly be clarified by exploration of the phenomenon in a larger number of genotypes and by carrying out a similar experiment with a population of RI derived from two different parental lines.

\section{Acknowledgements}

The authors are grateful to B. Burr for providing the RI lines and for critical reading of the manuscript. The skilful technical assistance of M. Villa is greatly appreciated. The research was supported by the National Research Council of Italy, Special Project RAISA, Sub-Project 2, Paper number 1150.

\section{References}

DAJOZ, I., TILL-BOTTRAUD, 1. AND GOUYON, P. H. 1992. Evolution of pollen morphology: the hare and tortoise game. In: Ottaviano E., Mulcahy D. L., Sari-Gorla M. and Bergamini Mulcahy G. (eds) Angiosperm Pollen and Ovules, pp. 420-425. Springer-Verlag, New York.

hormaza, J. I. AND HERrero, M. 1992. Pollen selection. Theor. Appl. Genet., 83, 663-672.

KINDIGER, B., BECKETT, T. A. AND COE, E. H. 1991. Differential effects of specific chromosomal deficiencies on the development of the maize pollen grain. Genome, 32, 579-594.

LINSKENS, H. F. AND PFAHLER, P. L. 1977. Genotypic effects on the amino acid relationship in maize (Zea mays L.) pollen and style. Theor. Appl. Genet., 50, 173-177.

MASCARENHAS, J. P. 1990 . Gene activity during pollen development. Ann. Rev. Plant Physiol. Plant Mol. Biol., 41, 317-338.

MEINKE, D. W. 1982. Embryo-lethal mutants of Arabidopsis thaliana. Evidence for gametophytic expression of the mutant genes. Theor. Appl. Genet., 63, 381-386.

ottaViano, E. AND MULCAHY, D. L. 1989. Genetics of Angiosperm pollen. Adv. Genet., 26, 1-64.

ottaViano, E., PETronl, D. AND PÉ, M. E. 1988. Gametophytic expression of genes controlling endosperm development in maize. Theor. Appl. Genet., 75, 252-258.

PFAHLER, P. L. 1967. Fertilization ability of maize pollen grains. II. Pollen genotype, female sporophyte and pollen storage interaction. Genetics, 57, 513-521.

SARI-GORLA, M., OtTaViano, E. AND Bellintani, R. 1976. Competitive ability of maize pollen. Interaction between genotypes of pollen and stylar tissues. Maydica, 21, 77-80.

SARI-GORLA, M., PE, M. E., MULCAHY, D. L. AND OTTAVIANO, E. 1992. Genetic dissection of pollen competitive ability in maize. Heredity, 69, 423-430. 